Transient musical hallucinations in a young adult male associated with alcohol withdrawal

Mao Lim ${ }^{1 *}$, Graham Blackman ${ }^{2}$, Anthony David ${ }^{3}$ and Fahmida Mannan ${ }^{2}$

${ }^{1}$ Cambridge University Hospitals NHS Foundation Trust;

${ }^{2}$ Department of Psychosis Studies, Institute of Psychiatry, Psychology and Neuroscience, King's College London and ${ }^{3}$ Institute of Mental

Health, University College London

${ }^{*}$ Corresponding author.

doi: 10.1192/bjo.2021.710

Aims. We present the case of a 25 -year-old male who presented to $A \& E$ with isolated musical hallucinations, in the absence of audiological or neurological disease.

Background. Musical hallucinations $(\mathrm{MH})$ are a form of complex auditory hallucinations whereby an individual experiences an instrumental and/or vocal melody in the absence of auditory stimuli.

Result. The patient had a history of recreational drug use and a family history of psychosis. Hallucinations, which were preceded by discontinuation of alcohol and re-initiation of citalopram for depression, resolved spontaneously after three days.

Conclusion. Aetiological factors are discussed alongside the existing literature. Whilst the underlying mechanisms underpinning musical hallucinations remains elusive, the case illustrates the potential role of alcohol withdrawal, serotonin toxicity, recreational drug use and genetic vulnerability.

\section{Development of a co-produced tool for monitoring and supporting the mental health of young people}

Joy MacKeith*, Anna Good and Sara Burns

Triangle

${ }^{\star}$ Corresponding author.

doi: 10.1192/bjo.2021.711

Aims. The aims were to develop and validate a tool for monitoring and supporting the mental health of young people. Based on extensive experience of developing similar tools, the hypothesis was that a user-friendly tool could be produced with sound psychometric properties.

Background. The Outcomes Star is a suite of collaboratively completed, strengths-based tools with the dual roles of both supporting and monitoring change. Service users are empowered through their active involvement in identifying their strengths and creating their care plan. Triangle, the creators of the Outcomes Star was approached by a number of organisations to develop a version of the Star for young people with mental health issues in early intervention services and also to support young people in managing a diagnosed mental illness.

Method. Using a series of focus groups and an iterative process of refinement we gathered data from practitioners and service users on the domains in which they wish to create change, and the steps of the change process. A draft version of the new tool was piloted in two organisations by 67 workers and 177 young people over six months. The pilot data were analysed to assess the psychometric properties of My Mind Star (acceptability, skew, factor structure, internal consistency, item redundancy and responsiveness).

Result. The resulting tool, My Mind Star consisted of seven domains: Feelings and emotions, Healthy lifestyle, Where you live, Friends and relationships, School, training and work, How you use your time and Self-esteem. Almost all young people and practitioners (94\%) agreed that their completed Star was 'a good summary of my life right now' and that it gave a better idea of service users' support needs. Psychometric analyses indicated a unidimensional structure with good internal consistency $(\alpha=.76)$ and no item redundancy. My Mind Star was responsive to change between the first and second readings, with medium and small-medium effect sizes.

Conclusion. Initial findings suggest that My Mind Star has good psychometric properties and is perceived as acceptable and useful by young people and practitioners, Further research is planned to conduct a full validation of the psychometric properties of this Star including inter-rater reliability and predictive validity.

Financial sponsorship of the study: Action for Children

The association between obesity and depression in adults: a meta-review

\author{
Abdi Malik Musa*, Samuele Cortese and Olivia Bloodworth \\ University of Southampton \\ ${ }^{\star}$ Corresponding author.
}

doi: $10.1192 /$ bjo.2021.712

Aims. Obesity and depression are increasing in prevalence and have become key issues in the public health of the modern day. We performed a meta-review to summarise the association between obesity and depression in adults.

Method. A systematic literature search was undertaken on MEDLINE, PsychINFO, EMBASE and Web of Science for systematic reviews (SRs) with or without meta-analyses (MA) on the association between obesity and depression in adults $(>18$ years) published before 18 September 2018. Any approach to define depressive disorders (e.g. via structured interview or code in medical file) was accepted. Likewise, any method to assess obesity was accepted. Screening, data extraction and quality assessment was completed by two reviewers independently, with a third reviewer to arbitrate any disagreement. AMSTAR 2 tool was used to assess the methodological quality and risk of bias of the pertinent SRs/MAs.

Result. After duplicate removal, we identified 6007 potentially pertinent citations. Following, title, abstract and full-text screening, 10 studies were included in the review; nine SRs with MAs and one SR. A statistically significant association between obesity and depression was reported in all nine SRs with MAs, with odds ratios ranging from $1.18(95 \% \mathrm{CI}=1.11-1.26)$ to $1.57(95 \% \mathrm{CI}=$ 1.53-2.01). Increased severity of obesity (body mass index over 40) was associated with a greater odds of becoming depressed. Odds of developing depression were greater for obese females, compared to obese males, but this difference was not statistically significant. Depression was shown to be a significant risk factor for future obesity in all four relevant MAs with odds ratios ranging from $1.18(95 \% \mathrm{CI}=1.13-1.23)$ to $1.40(95 \% \mathrm{CI}=1.14-1.71)$. Depressed adolescent females had the highest odds of becoming obese, significantly more so than depressed adolescent males and depressed adults. The quality of the included studies were mixed with five scoring moderate quality, three low quality and two critically low quality.

Conclusion. The findings suggest a reciprocal association between depression and obesity, which may be modulated by age and gender. Future research should assess the potential effect of obesity and depression severity more carefully while also exploring the underlying mechanisms. These results warrant the investigation of the effect of obesity or depression intervention on the outcomes of the other.

FUNDING

This research received no financial sponsorship. 
The outcome of an attachment-based infant mental health therapeutic play programme on infant temperament, parent-infant relationship \& maternal reflective functioning

Joy Malinit*

Philippine Children's Medical Center, University of the East Ramon Magsaysay Memorial Medical Center

${ }^{*}$ Corresponding author.

doi: 10.1192/bjo.2021.713

Aims. In the Philippines, there is a need for preventive, early intervention programs for perinatal and infant mental health. This is the first local study that investigated an attachment-based, therapeutic play programme (Baby Bonding) on infant temperament, parent-infant relationship and maternal reflecting functioning.

Background. This study was an effort towards bridging the "10/90 gap in infant mental health research" wherein $90 \%$ of the world's infants are born in low- middle-income countries (Population Reference Bureau, $2013 \mathrm{~b}$ ) and "only $10 \%$ of the worldwide spending on health research is directed towards the problems that primarily affect the poorest $90 \%$ of the world's population (Tomlinson et al., 2014).

Method. Phase I involved local validation of the Parent-Rated Outcome Measures (PROM)- Infant Characteristics Questionnaire (ICQ), Mother Object Relations Scale (MORS) and Parental Reflective Functioning Questionnaire (PRFQ).Healthy motherbaby dyads, from the low socio-economic stratum, were screened using Parent Evaluation of Developmental Status (PEDS) and Hospital and Anxiety Depression Scale (HADS).

Phase II carried out randomized controlled design wherein mother-baby dyads were enrolled either in the usual care group or the 6-weekly Baby Bonding intervention.

Result. 102 mothers answered the PROM. Their responses constituted the training set of the study tools. Baseline responses of the mothers from the usual care $(\mathrm{N}=51)$ and intervention $(\mathrm{N}=53)$ groups formed the evaluation set for the Filipino- translated instruments. In both the training and evaluation sets, if certain identified questions were to be removed from the PROM, better and acceptable Cronbach values were consistently generated.

There were no statistical differences on parent-infant relationship and reflective functioning between the usual care and intervention group. There was movement of the intervention group from high-challenging onto intermediate to low-levels of challenging temperament in the dull-dimension of the ICQ after 6 sessions. In comparison, infants in the control group remained in the high-challenging temperament after 6 weeks of usual care.

Conclusion. Linguistically validated study instruments (ICQ and MORS) provided accurate assessments of infant temperament and parent-child relationship. The Filipino-translated PRFQ has limited validity in evaluating parental reflective functioning (RF). "On-line" measures that video mother-baby interactions could have better captured changes in RF. As measured by the dull dimension of the ICQ, the Baby Bonding programme improved sociability of the infants ( 7 months or younger).

\section{Maternal immune activation generates anxiety in offspring: A translational meta-analysis}

Ursula Matos $^{1 \star}$, Laiana Azevedo Quagliato ${ }^{1}$ and Antonio Egidio Nardi ${ }^{2}$

${ }^{1}$ Institute of Psychiatry, Federal University of Rio de Janeiro and ${ }^{2}$ Full Professor of Psychiatry, Federal University of Rio de Janeiro ${ }^{\star}$ Corresponding author.

doi: 10.1192/bjo.2021.714
Aims. Maternal immune activation (MIA) during pregnancy is recognized as an etiological risk factor for various psychiatric disorders, such as schizophrenia, major depressive disorder, and autism. Prenatal immune challenge may serve as a "disease primer" into an altered trajectory of fetal brain development that, in combination with other genetic and environmental factors, may ultimately result in the emergence of different psychiatric conditions. However, the association between MIA and the offspring's chances of developing anxiety disorders is less clear. To examine the effect of MIA on offspring anxiety, a systematic review and meta-analysis of the preclinical literature was conducted.

Method. A systematic search of the PubMed, Web of Science, PsycINFO, and Cochrane Library electronic databases was performed using the PRISMA and WHO methodologies for systematic reviews. Studies that investigated if MIA during rodent's pregnancy could cause anxiety symptoms in offspring were included.

Result. Overall, the meta-analysis showed that MIA induced anxiety behavior in offspring. The studies provide strong evidence that prenatal immune activation impacts specific molecular targets, synapse formation and function, and a disbalance in neurotransmission that could be related to the generation of offspring anxiety. Future research should further explore the role of MIA in anxiety endophenotypes.

Conclusion. According to this meta-analysis, MIA plays an important role in the pathophysiological mechanisms of anxiety disorders and provides a promising therapeutic target.

\section{Exploring the mental health impact on private flat} owners in residential buildings with external combustible cladding

\section{William Martin*}

Warrington \& Halton NHS Trust, UK Cladding Action Group ${ }^{\star}$ Corresponding author.

\section{doi: 10.1192/bjo.2021.715}

Aims. To explore the mental health of private flat owners in residential buildings with external combustible cladding, which require remediation following the 2017 Grenfell tower fire.

The speed at which the fire at Grenfell spread, killing 72 people, is largely attributed to external combustible cladding. It is increasingly suspected that there are ongoing profound effects on the mental health of people living in flats with external combustible cladding like Grenfell both in the UK and abroad. The issue has left flat owners facing severe financial hardship, the threat of bankruptcy and concerns about safety in their own homes.

Method. An exploratory 'Google Forms' online mental health survey comprising multiple choice and free text questions over 47 sections was distributed to flat owners in affected buildings. The survey remained open for 6 weeks to allow response. 550 individual responses were studied.

Result. 550 individuals completed the survey, from 143 buildings across 45 UK councils.

As a direct result of external combustible cladding:

89.5\% said their mental health had deteriorated,

$22.5 \%$ reported having suicidal feelings or a desire to self-harm, $71.1 \%$ reported having difficulty sleeping,

$93.8 \%$ said they were suffering from worry and anxiety,

$59.6 \%$ used coping strategies to deal with their situation,

$35.1 \%$ said that existing physical and mental health conditions

had been exacerbated,

$84.1 \%$ said they cannot move on with their lives and 